\title{
Consistency of fat mass-fat-free mass relationship across ethnicity and sex groups
}

\author{
Stephanie T. Broyles*, Claude Bouchard, George A. Bray, Frank L. Greenway, William D. Johnson, \\ Robert L. Newton Jr, Eric Ravussin, Donna H. Ryan, Steven R. Smith and Peter T. Katzmarzyk \\ Pennington Biomedical Research Center, Louisiana State University System, 6400 Perkins Road, Baton Rouge, \\ LA 70808-4124, USA
}

(Received 28 June 2010 - Revised 11 October 2010 - Accepted 19 October 2010 - First published online 14 December 2010)

\begin{abstract}
The model developed by Forbes (1987) of how body fat mass (FM) and fat-free mass (FFM) change during periods of weight loss or gain ( $\Delta$ body weight $(\mathrm{BW}))$ assumed that they change in relationship to a constant $C=10 \cdot 4$, where $\Delta \mathrm{FFM} / \Delta \mathrm{BW}=10 \cdot 4 /(10 \cdot 4+\mathrm{FM})$. Forbes derived $C$ based on aggregated, cross-sectional data from a small sample of women. The objective of the present study was to reanalyse the relationship described by Forbes and to explore whether this relationship is consistent across ethnicity and sex groups using crosssectional data from a large sample of white and African-American men and women. Baseline data from white and African-American men and women aged 18-60 years, who participated in a clinical study at the Pennington Biomedical Research Center since 2001 and who underwent dual-energy X-ray absorptiometry scans, were available for analysis. To overcome differences in BMI distributions among the ethnicity-by-sex groups, a stratified random sample of participants was selected within each group such that numbers in each BMI category $\left(<25,25-29 \cdot 9,30-34 \cdot 9,35-39 \cdot 9,40+\mathrm{kg} / \mathrm{m}^{2}\right)$ were proportional to those within the group with the smallest sample size, yielding a sample of 1953 individuals. Linear regression models assessed the FM-FFM relationship across the four ethnicity-by-sex groups. The FM-FFM relationship varied little by ethnicity $(P=0 \cdot 57)$ or by sex $(P=0 \cdot 26)$. The constant describing the FM-FFM relationship was estimated to be $9 \cdot 7$ (95\% CI 9.0, 10.3). In conclusion, results from our large, biethnic sample of men and women found a FM-FFM relationship very close to that originally described by Forbes, absent of significant variability by ethnicity or sex.
\end{abstract}

\section{Key words: Body composition: Obesity: Weight loss}

Based on cross-sectional data on 164 women, Forbes ${ }^{(1)}$ derived a relationship between body fat mass (FM) and fatfree mass (FFM), which he used to make predictions about how these components of a two-compartment body composition model would change during periods of weight loss or gain. Forbes related FM and FFM using the following equation: $\quad F F M=14 \cdot 2+10 \cdot 4 \ln (\mathrm{FM})$. By differentiating and rearranging the equation, he observed that the crosssectional relationship could be used to predict the expected proportion of weight lost or gained as FFM, $\Delta \mathrm{FFM}$ / $\Delta \mathrm{BW}=10 \cdot 4 /(10 \cdot 4+\mathrm{FM})$, or conversely as $\mathrm{FM}, \Delta \mathrm{FM} /$ $\Delta \mathrm{BW}=\mathrm{FM} /(10 \cdot 4+\mathrm{FM})$, where $\Delta \mathrm{BW}$ indicates the amount of body weight lost or gained. Forbes' work suggested that the proportion of weight lost or gained as FM or FFM depends only on a person's initial FM and is consistent across weight loss modality (i.e. diet $v$. exercise) ${ }^{(2-4)}$, and that the relationship between FM and FFM during weight change could be described by a constant, $C=10 \cdot 4$ (Forbes' constant). The value of this constant indicates the predicted FM at which a person would lose or gain FM and FFM in equal amounts: persons having more than $10.4 \mathrm{~kg}$ of FM are expected to lose or gain a higher proportion of body weight as FM, and persons with less than $10.4 \mathrm{~kg}$ of FM are expected to lose or gain a higher proportion of body weight as FFM. This constant has been included in recent models that predict body composition changes and related changes in energy intake needs, following periods of weight loss ${ }^{(5)}$.

Forbes derived his original equation from cross-sectional data on 164 women aged 14-50 years, with body weights ranging from 28 to $147 \mathrm{~kg}$ and $\mathrm{FM}$ from 2 to $90 \mathrm{~kg}$ (estimated by whole-body ${ }^{40} \mathrm{~K}$ counting) ${ }^{(1)}$. The data were aggregated into five data points corresponding to body-fat-level groupings and analysed as such. We revisit Forbes' original analysis using individual-level (i.e. disaggregated) crosssectional data that represent both white and AfricanAmerican men and women and that do not include adolescents. We reanalyse the relationship described by

Abbreviations: FM, fat mass; FFM, fat-free mass.

*Corresponding author: Assistant Professor S. T. Broyles, fax +1 225763 3119, email stephanie.broyles@pbrc.edu 
Forbes and explore whether the cross-sectional FM-FFM relationship is similar across ethnicity and sex groups.

\section{Subjects and methods \\ Sample}

The Pennington Center Longitudinal Study is an ongoing investigation of obesity, lifestyle and the development of common chronic diseases based on a biethnic cohort of individuals who have participated in clinical studies at the Pennington Biomedical Research Center since 1992. The present analysis considers baseline (cross-sectional) data from participants aged 60 years or less who underwent dual-energy X-ray absorptiometry scans (before any interventions) using a whole-body scanner, Hologic QDR 4500A (Bedford, MA, USA), which has been used since 2001. The sample includes 2709 adults aged 18-60 years (mean 40.4 years), with 953 white women, 790 African-American women, 708 white men and 258 African-American men. The present study was conducted according to the guidelines laid down in the Declaration of Helsinki, and all procedures involving human subjects/ patients were approved by the Pennington Biomedical Research Center Institutional Review Board. Written informed consent was obtained from all subjects/patients.

\section{Anthropometry and dual-energy X-ray absorptiometry}

During each volunteer's initial study visit, a nurse measured height in duplicate using a wall-mounted stadiometer and weight in duplicate using a digital scale after the volunteer removed outer clothing, heavy pocket items and shoes. BMI was calculated as weight (kg)/ height $\left(\mathrm{m}^{2}\right)$. Total body FM $(\mathrm{kg})$ and FFM (kg) were estimated by dual-energy X-ray absorptiometry using a Hologic QDR 4500A whole-body scanner.

\section{Statistical analysis}

Data management and analysis were performed using Statistical Analysis Systems Statistical software (version 9.1; SAS Institute, Cary, NC, USA). Differences in the relationship between FM and FFM across age, sex and ethnicity groups were analysed by regression analyses. FFM was adjusted for height $\left(\text { height }^{2}\right)^{(6)}$ for two reasons: (1) Forbes' original work controlled for height via inclusion criteria, while no such restrictions are imposed on our sample; (2) Forbes' work and extensions seek to explain how FM and FFM change in relation to each other over time for an individual, i.e. in a situation where $\triangle F F M$ would be controlled for height. In our sample, FFM was related to height $\left(r^{2}\right.$ 0.59). FM was log-transformed, based on Forbes' empirical observations ${ }^{(1,4)}$ and on other evidence $^{(7)}$ suggesting that FFM appears to be a logarithmic function of FM over the range of possible FM values.
Forbes' analysis considered the relationship between $\ln$ (FM) and FFM to be constant across all levels of FM (i.e. linear regression with constant slope), and the present analysis replicates Forbes' original analysis in terms of this assumption. Forbes derived his constant $C=10.4$ from the slope of the $\ln$ (FM)-FFM relationship in his crosssectional sample. Similarly, the present analysis estimates this regression coefficient and compares it with Forbes' value of 10.4. Except where noted, separate linear regression models were fit for each ethnicity-by-sex group.

\section{Adjustment for ethnicity-sex differences in BMI distribution}

Forbes' original analysis considered the FM-FFM relationship to be constant over the entire range of FM levels. This observation has not been tested in other samples and is not considered in the present study. However, we observed that the BMI distributions differed considerably among the four ethnicity-by-sex groups in our sample; therefore, to avoid any potential bias in our comparisons of the FM-FFM relationship across ethnicity and sex, we selected a stratified random sample of participants within each group such that the numbers in each BMI category $(<25$, $25-29 \cdot 9,30-34 \cdot 9,35-39 \cdot 9,40+\mathrm{kg} / \mathrm{m}^{2}$ ) were proportional to those within African-American men, the group with the smallest overall sample size. All subsequent analyses were conducted on this random sample ( $n$ 1953). The common adjustment approach of including BMI category as a covariate in our regression models was not possible, as this strategy, in effect, describes the 'within BMI category' relationship between FM and FFM, which is constrained to be negative due to how BMI is calculated (i.e. for a given range of BMI values, FM must decrease as FFM increases in order to have the BMI value remain in the specified range), rather than the relationship described by Forbes.

\section{Results}

The sample on which Forbes based his relationship was composed of 164 women aged 14-50 years, with body weight ranging from 28 to $147 \mathrm{~kg}$ and FM from 2 to $90 \mathrm{~kg}$ (estimated by ${ }^{40} \mathrm{~K}$ assays) ${ }^{(1)}$. Compared with Forbes' sample, ours includes men, is older, does not include persons under the age of 18 years, and contains a smaller proportion of underweight individuals (Forbes' sample included twelve females with anorexia nervosa) (Table 1). Additionally, Forbes controlled for height by limiting his sample to women between heights of 156 and $170 \mathrm{~cm}^{(1)}$, while our sample contains a wider height range (women: 143-184 cm; men: $154-198 \mathrm{~cm}$ ). In our sample, FM ranged from 4.6 to $75.4 \mathrm{~kg}$, and FFM ranged from $30 \cdot 1$ to $100 \cdot 1 \mathrm{~kg}$ The prevalences of normal weight, overweight and obesity were $16.5,25.8$ and $57.7 \%$, respectively. Although not shown, age, height and ranges for BMI, FM and FFM were similar between the full sample of Pennington Center 
Table 1. Descriptive characteristics of sample, by sex and ethnicity

(Mean values, standard deviations and ranges)

\begin{tabular}{|c|c|c|c|c|c|c|c|c|c|c|c|c|}
\hline & \multicolumn{6}{|c|}{ Men } & \multicolumn{6}{|c|}{ Women } \\
\hline & \multicolumn{3}{|c|}{ White } & \multicolumn{3}{|c|}{ African-American } & \multicolumn{3}{|c|}{ White } & \multicolumn{3}{|c|}{ African-American } \\
\hline & Mean & SD & Range & Mean & $\mathrm{SD}$ & Range & Mean & SD & Range & Mean & SD & Range \\
\hline$n$ & \multicolumn{3}{|c|}{568} & \multicolumn{3}{|c|}{258} & \multicolumn{3}{|c|}{710} & \multicolumn{3}{|c|}{417} \\
\hline Age (years) & $39 \cdot 6$ & $12 \cdot 9$ & $18-60$ & 34.9 & 12.9 & $18-60$ & 42.9 & 11.5 & $18-60$ & $36 \cdot 6$ & 11.5 & $18-60$ \\
\hline Height (cm) & $177 \cdot 7$ & $6 \cdot 4$ & $154-198$ & $177 \cdot 4$ & $6 \cdot 9$ & $155-198$ & $163 \cdot 4$ & $6 \cdot 0$ & $144-184$ & $163 \cdot 4$ & $6 \cdot 3$ & $148-180$ \\
\hline Weight (kg) & $94 \cdot 0$ & $17 \cdot 9$ & $56 \cdot 3-163 \cdot 3$ & $94 \cdot 0$ & $18 \cdot 3$ & $55 \cdot 0-148 \cdot 9$ & $79 \cdot 0$ & $16 \cdot 0$ & $42 \cdot 1-133 \cdot 1$ & $79 \cdot 6$ & $15 \cdot 7$ & $41 \cdot 5-134 \cdot 7$ \\
\hline BMI $\left(\mathrm{kg} / \mathrm{m}^{2}\right)$ & $29 \cdot 8$ & $5 \cdot 4$ & $18 \cdot 1-46 \cdot 0$ & $29 \cdot 8$ & 5.6 & $18 \cdot 3-49 \cdot 0$ & $29 \cdot 6$ & $5 \cdot 7$ & $17 \cdot 2-49 \cdot 4$ & $29 \cdot 8$ & 5.5 & $17 \cdot 3-49 \cdot 8$ \\
\hline \multicolumn{13}{|l|}{ BMI categories (\%) } \\
\hline$<25 \mathrm{~kg} / \mathrm{m}^{2}$ & \multicolumn{3}{|c|}{21} & \multicolumn{3}{|c|}{21} & \multicolumn{3}{|c|}{21} & \multicolumn{3}{|c|}{21} \\
\hline $25 \cdot 0-29 \cdot 9 \mathrm{~kg} / \mathrm{m}^{2}$ & \multicolumn{3}{|c|}{31} & \multicolumn{3}{|c|}{31} & \multicolumn{3}{|c|}{31} & \multicolumn{3}{|c|}{31} \\
\hline $30.0-34.9 \mathrm{~kg} / \mathrm{m}^{2}$ & \multicolumn{3}{|c|}{31} & \multicolumn{3}{|c|}{31} & \multicolumn{3}{|c|}{31} & \multicolumn{3}{|c|}{31} \\
\hline $35.0-39.9 \mathrm{~kg} / \mathrm{m}^{2}$ & \multicolumn{3}{|c|}{13} & \multicolumn{3}{|c|}{13} & \multicolumn{3}{|c|}{13} & \multicolumn{3}{|c|}{13} \\
\hline$\geq 40.0 \mathrm{~kg} / \mathrm{m}^{2}$ & \multicolumn{3}{|c|}{3} & \multicolumn{3}{|c|}{3} & \multicolumn{3}{|c|}{3} & \multicolumn{3}{|c|}{3} \\
\hline Fat mass (kg) & $25 \cdot 1$ & $11 \cdot 0$ & $5 \cdot 6-66 \cdot 0$ & $22 \cdot 6$ & $11 \cdot 0$ & $4 \cdot 6-60 \cdot 9$ & 30.9 & $10 \cdot 7$ & $6 \cdot 8-66 \cdot 9$ & $30 \cdot 0$ & $10 \cdot 1$ & $8 \cdot 2-69 \cdot 8$ \\
\hline Fat-free mass (kg) & $69 \cdot 8$ & $8 \cdot 8$ & $46 \cdot 1-100 \cdot 1$ & 71.4 & 9.6 & $47 \cdot 1-96 \cdot 8$ & $48 \cdot 2$ & $6 \cdot 7$ & $30.5-75.9$ & $49 \cdot 7$ & $7 \cdot 1$ & $30 \cdot 1-82 \cdot 1$ \\
\hline
\end{tabular}

Longitudinal Study participants with dual-energy X-ray absorptiometry and the random sample ( $n$ 1953) to which the regression models were applied.

In regression models of $\ln (\mathrm{FM}) v$. FFM, the predicted slopes of the $\ln (\mathrm{FM})-\mathrm{FFM}$ relationship (corresponding to the constant $C$ derived in Forbes' original analysis) ranged from $9 \cdot 2$ (white men) to $10 \cdot 4$ (African-American women). Men and African-Americans had a higher amount of FFM for a given FM $(P<0 \cdot 0001$, by sex comparison; $P<0 \cdot 0001$, by ethnicity comparison); however, the slopes of the $\ln$ (FM)-FFM relationship did not differ significantly by sex $(P=0 \cdot 26)$ or by ethnicity $(P=0.57)$ (Table 2 and Fig. 1$)$. When data were pooled across ethnicity-by-sex groups, the predicted slope describing the relationship between $\ln$ (FM) and FFM was $9 \cdot 7$ (95\% CI 9.0, 10.3; Table 2).

Forbes' constant $C$ is used to predict the proportion of weight lost or gained as fat for any given FM (Fig. 2).
The constant derived from our sample yields a prediction curve very close to that based on Forbes' value of $C=10 \cdot 4$.

\section{Discussion}

Forbes derived a relationship that predicted how FM and FFM would change during periods of weight loss or gain $^{(1,4)}$. Although his analysis was based on crosssectional data, using the derivative of the cross-sectional relationship, Forbes attempted to explain how body composition changed with weight change due to either nutrition or exercise. Central to this relationship was a constant, $C=10 \cdot 4$, that indicates the predicted FM at which a person would lose or gain FM and FFM in equal amounts: persons having more than $10.4 \mathrm{~kg}$ of FM are expected to lose or gain a higher proportion of body weight as FM, and persons with less than $10 \cdot 4 \mathrm{~kg}$ of FM are expected to

Table 2. Summary of regression analyses evaluating the relationship between fat mass (FM) and fat-free mass (FFM) across levels of FM, by sex and ethnicity

( $b$ Coefficients and $95 \%$ confidence intervals)

\begin{tabular}{|c|c|c|c|c|}
\hline \multirow[b]{2}{*}{ Model } & \multicolumn{2}{|r|}{ Men } & \multicolumn{2}{|r|}{ Women } \\
\hline & White ( $n 568)$ & African-American ( $n$ 258) & White ( $n 710)$ & African-American ( $n$ 417) \\
\hline \multicolumn{5}{|c|}{ Separate models for each ethnicity-by-sex group* } \\
\hline $\begin{array}{l}\text { Estimated FFM at } F M=20 \mathrm{~kg} \dagger \\
\ln (\mathrm{FM}) \ddagger\end{array}$ & 67.5 & $71 \cdot 6$ & $44 \cdot 7$ & $46 \cdot 0$ \\
\hline$b$ & $9 \cdot 2$ & $9 \cdot 2$ & $9 \cdot 7$ & $10 \cdot 4$ \\
\hline $95 \% \mathrm{Cl}$ & $7 \cdot 9,10.5$ & $7 \cdot 7,10 \cdot 7$ & $8 \cdot 6,10 \cdot 7$ & $8 \cdot 9,12 \cdot 0$ \\
\hline $\begin{array}{l}r^{2} \\
\text { Single model§ }\end{array}$ & 0.35 & 0.42 & 0.31 & 0.31 \\
\hline $\begin{array}{l}\text { Estimated FFM at FM }=20 \mathrm{~kg} \\
\ln (\mathrm{FM})\end{array}$ & $67 \cdot 4$ & 71.5 & $44 \cdot 6$ & $46 \cdot 4$ \\
\hline$r^{2} \frac{b}{95 \% \mathrm{Cl}}$ & \multicolumn{4}{|c|}{$\begin{array}{c}9 \cdot 0,10 \cdot 3 \\
0.78\end{array}$} \\
\hline
\end{tabular}

* Separate regressions were conducted for each ethnicity-by-sex group. Model also adjusted for age.

†Ethnicity comparison $(P<0.0001)$; sex comparison $(P<0.0001)$.

$\ddagger$ Ethnicity comparison $(P=0.5733)$; sex comparison $(P=0.2606)$.

$\S$ Single regression was conducted. Model also adjusted for age, ethnicity, sex, and ethnicity $\times$ sex. 

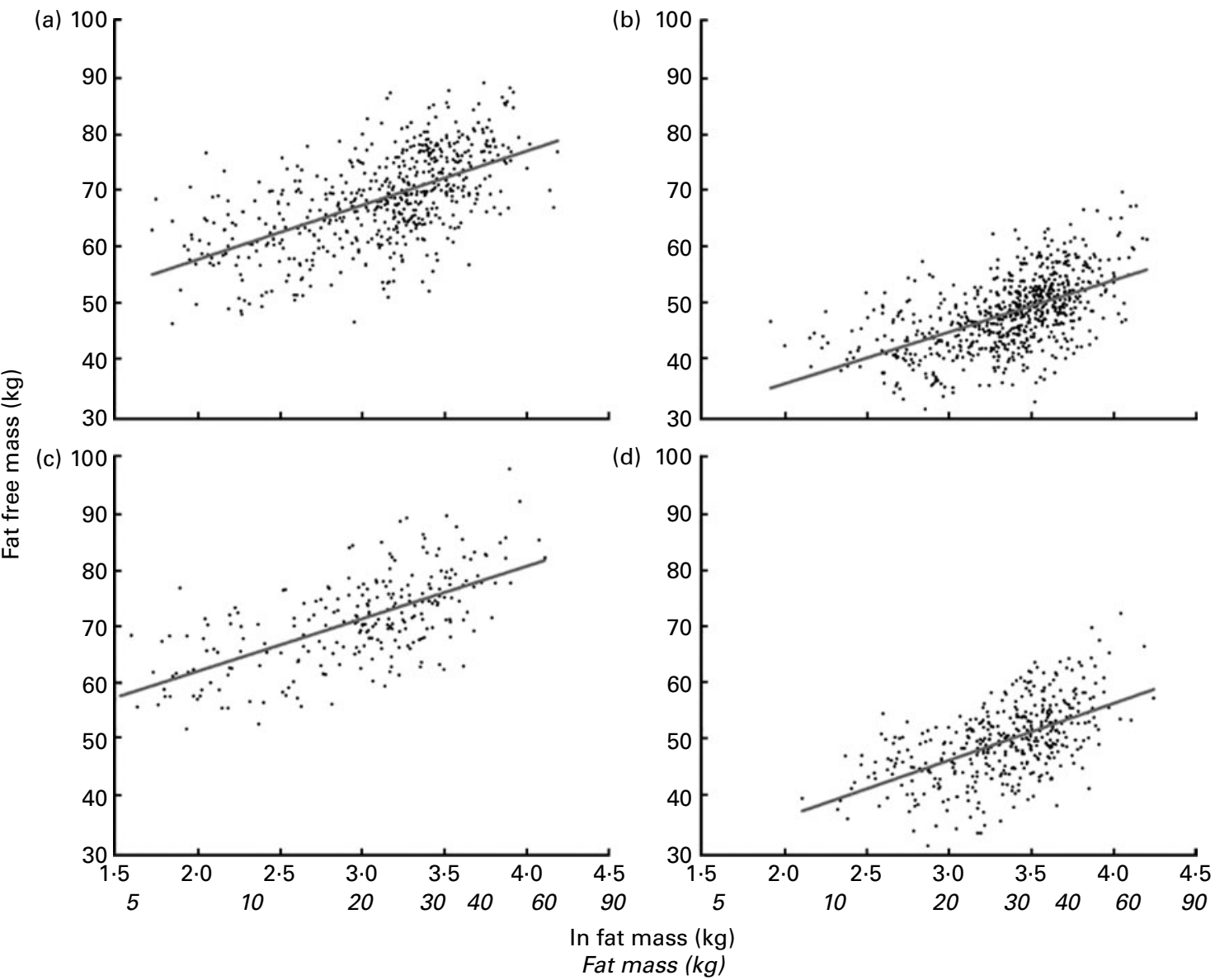

Fig. 1. Semi-log plot of fat mass $v$. fat-free mass (FFM) (points) and predicted levels of FFM ( - ), by ethnicity and sex: (a) white men, $b=9 \cdot 2$; (b) white women, $b=9 \cdot 7$; (c) African-American men, $b=9 \cdot 2$; (d) African-American women, $b=10.4$.

lose or gain a higher proportion of body weight as FFM. The present results suggest that this constant is consistent between men and women and between whites and African-Americans.

Despite the differences between our sample and that of Forbes and in the techniques used to assess body

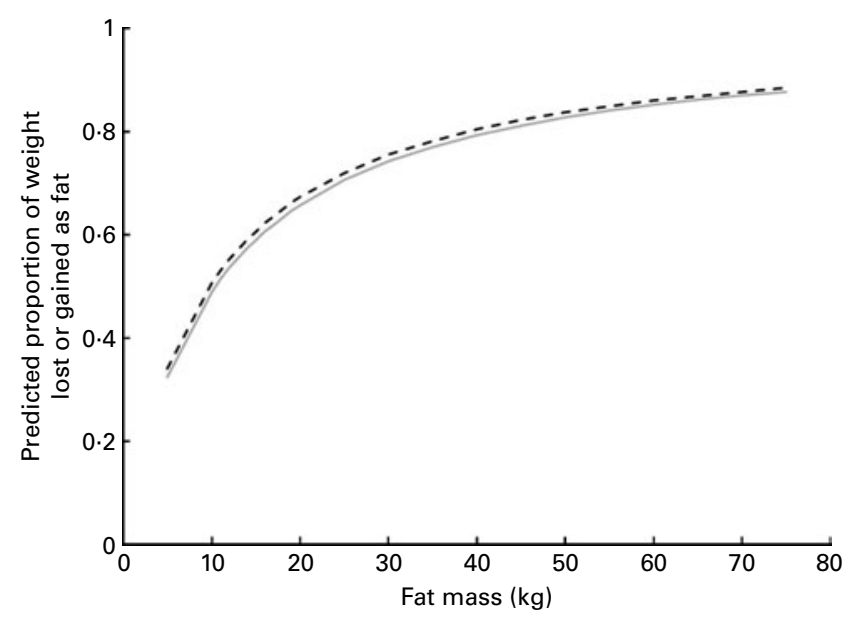

Fig. 2. Predicted proportion of weight lost or gained as fat according to estimated parameters of various models $(C)$ : $\_$Forbes' model $(C=10.4)$; - - - Pennington Center Longitudinal Study model $(C=9 \cdot 7)$. composition, the relationship derived in the present study yields predicted body composition changes very close to that of Forbes (Fig. 2). Over the range of FM values, Forbes' prediction of the proportion of weight lost or gained as fat differed from ours by less than 0.02 or $2 \%$.

The present finding that men and women are expected to lose similar proportions of weight as FM is inconsistent with observations from other studies (reviewed in Chaston et $\left.a l^{(8)}\right)$ that found a tendency for men to lose a lower proportion of weight as FM, compared with women. Few studies have reported changes in weight and FM by ethnicity; however, one such study found no differences in the amount of weight lost as FM between white and AfricanAmerican women ${ }^{(9)}$, which is consistent with the present findings of no overall effect of ethnicity.

The similarities that we found in the cross-sectional relationship between FM and FFM exist despite differences in the amount of FFM for a given amount of FM, with men and African-Americans having higher amounts of FFM than women and whites. These observed differences are consistent with those of others. Adult men, compared with women, have a higher FFM per unit stature, a higher skeletal muscle mass ${ }^{(10)}$ and a higher bone mineral content ${ }^{(11)}$. 
African-Americans, compared with whites, have a higher bone mineral content and density, a higher whole-body protein content and a relatively longer limb length (reviewed in Wagner \& Heyward ${ }^{(12)}$ ).

While these data build on Forbes' earlier work by analysing the cross-sectional relationship between FM and FFM in a large, biethnic sample of men and women, we have not challenged the assumption that within-individual longitudinal changes in FM and FFM proportions accompanying weight change follow the cross-sectional relationship described here nor even that the cross-sectional relationship is correctly specified by the Forbes model. It is possible that the inconsistencies observed between our predictions and results from weight loss studies ${ }^{(8)}$ are related to these issues. Moreover, Forbes' assertion that the FM:FFM ratio in a weight loss or gain situation is not influenced by the weight change modality, e.g. $\operatorname{diet} v$. exercise $\mathrm{e}^{(2-4)}$, is not consistently upheld in weight loss studies ${ }^{(13)}$. Further research using longitudinal study designs is needed to explore these questions.

In conclusion, results from a large, biethnic sample of men and women suggest that the FM-FFM relationship originally described by Forbes in women is consistent across ethnicity and sex. Future research should explore whether longitudinal body composition changes conform to the relationship predicted by cross-sectional data.

\section{Acknowledgements}

The present study was supported by the Pennington Biomedical Research Center. C. B. is funded, in part, by the George A. Bray, Jr, Chair of Nutrition; E. R. is funded, in part, by the Douglas L. Gordon Chair in Diabetes and Metabolism; P. T. K. is supported, in part, by the Louisiana Public Facilities Authority Endowed Chair in Nutrition. The authors made the following contributions to the present study - S. T. B. analysed the data and drafted the manuscript; C. B. and R. L. N. edited the manuscript; G. A. B., F. L. G., E. R., D. H. R. and S. R. S. collected the data and edited the manuscript; W. D. J. aided in data analysis and edited the manuscript; P. T. K. conceived of the study and edited the manuscript. None of the authors has a conflict of interest. The authors also acknowledge the clinical scientists and staff of the Pennington Biomedical Research Center who have contributed data to the Pennington Center Longitudinal Study, particularly William Cefalu, Nikhil Dhurandhar, Lilian Levitan, Timothy Church, Paula Geiselman, Alok Gupta, Corby Martin and Thomas Gettys.

\section{References}

1. Forbes GB (1987) Lean body mass-body fat interrelationships in humans. Nutr Rev 45, 225-231.

2. Forbes GB (1991) Exercise and body composition. J Appl Physiol 70, 994-997.

3. Forbes GB (1992) Exercise and lean weight: the influence of body weight. Nutr Rev 50, 157-161.

4. Forbes GB (2000) Body fat content influences the body composition response to nutrition and exercise. Ann $N Y$ Acad Sci 904, 359-365.

5. Hall KD \& Jordan PN (2008) Modeling weight-loss maintenance to help prevent body weight regain. Am J Clin Nutr $\mathbf{8 8}$, $1495-1503$.

6. Heymsfield SB, Gallagher D, Mayer L, et al. (2007) Scaling of human body composition to stature: new insights into body mass index. Am J Clin Nutr 86, 82-91.

7. Hall KD (2007) Body fat and fat-free mass inter-relationships: Forbes's theory revisited. Br J Nutr 97, 1059-1063.

8. Chaston TB, Dixon JB \& O'Brien PE (2007) Changes in fat-free mass during significant weight loss: a systematic review. Int J Obes (Lond) 31, 743-750.

9. Weinsier RL, Hunter GR, Schutz Y, et al. (2002) Physical activity in free-living, overweight white and black women: divergent responses by race to diet-induced weight loss. Am J Clin Nutr 76, 736-742.

10. Malina RM, Bouchard C \& Bar-Or O (2004) Growth, Maturation, and Physical Activity, 2nd ed. Champaign, IL: Human Kinetics.

11. Horber FF, Gruber B, Thomi F, et al. (1997) Effect of sex and age on bone mass, body composition and fuel metabolism in humans. Nutrition 13, 524-534.

12. Wagner DR \& Heyward VH (2000) Measures of body composition in blacks and whites: a comparative review. Am J Clin Nutr 71, 1392-1402.

13. Heymsfield SB (2010) Physical activity, weight loss, and maintenance of lean mass. In Physical Activity and Obesity [C Bouchard and PT Katzmarzyk, editors]. Champaign, IL: Human Kinetics. 